\title{
Serum osteopontin concentration is decreased by exercise-induced fat loss but is not correlated with body fat percentage in obese humans
}

\author{
JEONG SOON YOU ${ }^{1}$, HYE-IN JI ${ }^{2}$, KYUNG JA CHANG ${ }^{1}$, MYUNG CHUL YOO ${ }^{2}$, \\ HYUNG-IN YANG ${ }^{2}$, IN-KYUNG JEONG ${ }^{3}$ and KYOUNG SOO KIM ${ }^{2}$
}

\author{
${ }^{1}$ Department of Food and Nutrition, Inha University, Nam-gu, Incheon 402-751; \\ ${ }^{2}$ East-West Bone and Joint Disease Research Institute, and ${ }^{3}$ Department of Endocrinology, Kyung Hee University \\ Hospital at Gangdong, Kyung Hee University College of Medicine, Gangdong-gu, Seoul 134-727, Republic of Korea
}

Received February 16, 2013; Accepted June 11, 2013

DOI: $10.3892 / \mathrm{mmr} .2013 .1522$

\begin{abstract}
To evaluate the extent to which fat mass contributes to serum osteopontin (OPN) concentration, we investigated whether serum OPN levels are decreased by exercise-induced fat mass loss and whether they are associated with body fat percentage in obese humans. Twenty-three female college students were recruited to participate in an 8-week body weight control program. Body composition [body weight, soft lean mass, body fat mass, body fat percentage, waist-hip ratio and body mass index (BMI)] were assessed prior to and following the program. Serum lipid profiles and serum adiponectin, leptin and osteopontin levels were measured from serum collected prior to and following the program. To understand the effect of fat mass loss on the serum levels of adipokine, which is mainly produced in adipose tissue, the leptin and adiponectin levels were also measured prior to and following the program. Serum leptin levels (mean \pm standard error of the mean) decreased significantly following the program (from $9.82 \pm 0.98$ to $7.23 \pm 0.67 \mathrm{ng} / \mathrm{ml}$ ) and were closely correlated with body fat percentage. In addition, serum adiponectin levels were negatively correlated with body fat percentage, while serum adiponectin levels were not significantly altered. By contrast, serum OPN levels decreased significantly following the program (from $16.03 \pm 2.34$ to $10.65 \pm 1.22 \mathrm{ng} / \mathrm{ml}$ ). However, serum OPN levels were not correlated with body fat percentage, suggesting that serum OPN levels are controlled by several other factors in humans. In conclusion, a high expression of OPN in adipose tissues may not be correlated with serum OPN
\end{abstract}

Correspondence to: Professor Kyoung Soo Kim, East-West Bone and Joint Disease Research Institute, Kyung Hee University Hospital at Gangdong, Kyung Hee University College of Medicine, Gangdong-gu, Seoul 134-727, Republic of Korea

E-mail: labrea46@naver.com

Key words: osteopontin, adiponectin, leptin, obesity, exercise-induced fat mass loss levels in obese humans. Thus, tissues or physiological factors other than fat mass may have a greater contribution to the serum OPN levels.

\section{Introduction}

Adipose tissue is characterized by the infiltration of immune cells, such as macrophages and $\mathrm{T}$ cells, during obesity (1). Macrophages release cytokines, such as IL- $1 \beta$, IL- 6 and TNF- $\alpha$, leading to a proinflammatory environment. Furthermore, the immune cells in adipose tissues, including activated macrophages, $\mathrm{T}$ cells and dendritic cells (DCs), produce a pleiotropic cytokine, osteopontin (OPN), which is upregulated during inflammation (2). The obesity-driven inflammation and macrophage accumulation is blocked by OPN deficiency (3). OPN expression is significantly upregulated in the adipose tissue of high fat diet-induced and genetically obese mice, while it remains largely unaltered in the liver $(4,5)$. OPN acts as a chemokine and an inflammatory cytokine through a variety of different receptors, including CD44 and integrins. Thus, it is important in various inflammatory disorders, such as rheumatoid arthritis (6), experimental autoimmune encephalomyelitis (EAE), multiple sclerosis (MS) (7), allergic disease (8) and cardiovascular disease (9). It is also involved in non-inflammatory pathophysiological processes, including bone remodeling, neoplastic transformation, progression of metastases, promotion of cell survival and wound healing $(10,11)$. Therefore, OPN has been investigated as a therapeutic target for certain diseases $(12,13)$.

Serum OPN levels are positively correlated with body fat percentage and may be reduced by fat mass loss. Thus, diet-induced weight loss has been demonstrated to significantly decrease plasma OPN concentrations (5). This reduction of OPN was hypothesized to be secondary to the loss of adipose tissue. However, bariatric surgery, the most effective treatment to achieve weight loss in morbidly obese humans, has been identified to gradually increase plasma OPN levels, although it significantly reduced the body weight, body mass index (BMI), waist circumference, homeostasis model of assessment-insulin resistance and blood concentrations of 
Table I. Changes in the anthropometric parameters of the subjects before and after the 8-week body weight control program.

\begin{tabular}{lccc}
\hline Variables & Before & After & Difference \\
\hline Age (years) & $20.7 \pm 0.41$ & & \\
Height $(\mathrm{cm})$ & $161.1 \pm 1.3$ & $60.5 \pm 1.7$ & $-2.1 \pm 0.3^{\mathrm{a}}$ \\
Body weight $(\mathrm{kg})$ & $62.5 \pm 1.7$ & $23.3 \pm 0.7$ & $-0.8 \pm 0.1^{\mathrm{a}}$ \\
Body mass index $\left(\mathrm{kg} / \mathrm{m}^{2}\right)$ & $24.1 \pm 0.7$ & $39.3 \pm 1.0$ & $1.1 \pm 0.2^{\mathrm{a}}$ \\
Soft lean mass $(\mathrm{kg})$ & $38.2 \pm 1.0$ & $18.8 \pm 0.9$ & $-3.2 \pm 0.3^{\mathrm{a}}$ \\
Body fat mass $(\mathrm{kg})$ & $22.0 \pm 0.8$ & $30.9 \pm 0.9$ & $-4.0 \pm 0.4^{\mathrm{a}}$ \\
Percentage body fat $(\%)$ & $35.1 \pm 0.7$ & & \\
\end{tabular}

Values are expressed as the mean $\pm \mathrm{SEM} .{ }^{\mathrm{a}} \mathrm{P}<0.001$, by a paired t-test.

C-reactive protein $(14,15)$. An elevated OPN concentration following bariatric surgery and weight loss is hypothesized to reflect the increased bone turnover, which is secondary to the reduced weight load on bone. In addition, the plasma OPN concentration remained unchanged in murine models of obesity, regardless of an elevated expression of OPN in adipose tissue. Furthermore, OPN levels are only moderately altered in morbidly obese patients (4). Thus, whether serum OPN levels are associated with body fat percentage has not been elucidated in mice and humans. OPN is also produced by multiple tissues, including epithelia, kidney, thyroid, breast, uterus, placenta and testes (16). Furthermore, OPN is highly expressed in bone matrix (17) and is important in bone turnover, anchoring osteoclasts to bone and activating the resorption cascade (18). This may indicate that serum OPN levels are not only regulated by fat mass but also by other tissues. In the present study, to evaluate how significantly fat mass contributes to serum OPN concentrations, we investigated whether serum OPN levels are decreased by exercise-induced fat mass loss and are associated with body fat percentage in obese humans (excluding those that were morbidly obese).

\section{Subjects and methods}

Study subjects. Study subjects (23 female college students aged 19-23 years) were recruited from Inha University (Incheon, Korea). All 23 subjects submitted written informed consent to participate in an 8-week body weight control program. The study protocol was reviewed and approved by the Institutional Review Board at Kyung Hee University Hospital (Seoul, Korea). To investigate whether serum OPN levels are predominantly dependant on fat mass, only obese subjects $(n=23)(>30 \%$ body fat percentage), based on body fat percentage and not on BMI, were recruited. Out of the 23 subjects recruited, 18 completed the 8 -week body weight control program. Morbidly obese females were excluded, as excessive fat mass loss may overwhelm the effect of other tissues or factors on serum OPN concentration. Moreover, the low frequency of morbidly obese individuals suggests that subjects would be difficult to find and recruit.

The subjects were free-living and were allowed a self-selected diet. No medication or other nutritional supplements were taken. The study was conducted from May to July, 2010. The analysis of the results was conducted for the 18 students who completed the 8-week program (drop out rate, $\sim 21.7 \%$ ). The subjects did not present with any chronic diseases, and did not take any medication.

Body weight control program. The 8-week body weight control program consisted of diet therapy, exercise and behavioral change. The subjects were recommended by a dietitian at an introductory class to consume an individualized low-calorie diet. The subjects were required to perform treadmill exercise three times a week during the 8 -week program, reaching $70 \%$ of the anaerobic threshold (AT), in order to consume 200 kcal during exercise. To implement behavioral changes, subjects were provided with an online lecture and were asked to submit a weekly self-monitored diet and exercise diary to the researcher. In addition, subjects were counseled at weekly face-to-face meetings and via e-mail.

Body composition assessment. Anthropometric measurements were obtained from each subject. Individual height was measured with an anthropometer and body composition (body weight, soft lean mass, body fat mass, body fat percentage, waist-hip ratio and BMI) was assessed at least once per week using bioelectrical impedance (InBody 3.0, Biospace Co., Ltd., Seoul, Korea) (Table I).

Serum lipid profiles. Following overnight fasting, blood was collected at a specific time in the morning prior to and following the program. The collected blood was centrifuged at $1650 \mathrm{x} \mathrm{g}$ for $15 \mathrm{~min}$. The supernatant serum was separated in microtubes and stored at $-70^{\circ} \mathrm{C}$ until it was analyzed for the serum lipid concentrations. Serum total cholesterol (TC), high-density lipoprotein (HDL) cholesterol and triglyceride (TG) levels were determined using an automatic clinical analyzer (BPC BioSed Srl, Rome, Italy). Serum low-density lipoprotein (LDL) cholesterol levels were calculated from the serum TC, HDL and TG levels (19) (Table II).

Serum osteopontin, adiponectin and leptin levels. The collected sera were analyzed for osteopontin, adiponectin and leptin with an enzyme-linked immunosorbent assay (ELISA) kit according to the manufacturer's instructions ( R\&D Systems, Inc., Minneapolis, MN, USA).

Statistical analysis. Experimental data are expressed as the mean \pm standard error of the mean (SEM). The levels of fat 
Table II. Changes in the serum lipid profiles of the subjects before and after the 8-week body weight control program.

\begin{tabular}{lrrr}
\hline Serum lipid level $(\mathrm{mg} / \mathrm{dl})$ & Before & After & Difference \\
\hline Total cholesterol & $188.0 \pm 6.3$ & $178.0 \pm 4.5$ & $-9.7 \pm 3.5^{\mathrm{a}}$ \\
HDL cholesterol & $43.7 \pm 3.1$ & $41.5 \pm 2.7$ & $-1.3 \pm 1.2$ \\
LDL cholesterol & $125.9 \pm 5.6$ & $120.7 \pm 4.9$ & $-4.61 \pm 2.9$ \\
Triglyceride & $97.4 \pm 12.0$ & $68.9 \pm 8.6$ & $-28.2 \pm 4.7^{\mathrm{b}}$ \\
\hline
\end{tabular}

Values are expressed as the mean \pm SEM. ${ }^{a} \mathrm{P}<0.05$ and ${ }^{\mathrm{b}} \mathrm{P}<0.01$, by a paired t-test. HDL, high-density lipoprotein; LDL, low-density lipoprotein

and muscle mass and serum adiponectin, leptin and osteopontin prior to and following the 8-week body weight control program were compared using a Wilcoxon signed-rank test (two-tailed). To determine the degree of linearity between two variables, the data were compared using the Spearman's correlation test (two-tailed). Prism software, version 5.02 (Graphpad Software Inc., La Jolla, CA, USA) was used for statistical analysis and graphing. $\mathrm{P}<0.05$ was considered to indicate a statistically significant difference.

\section{Results}

Effect of the 8-week exercise program on fat and muscle mass. Following the program, the average BMI decreased significantly from $24.1 \pm 0.7$ to $23.3 \pm 0.7 \mathrm{~kg} / \mathrm{m}^{2}$, as did the average body fat percentage, from $35.1 \pm 0.7$ to $30.9 \pm 0.9 \%$, respectively (Table I). Consistent with the BMI and body fat percentage reductions, the fat and muscle masses were significantly decreased and increased, respectively (Fig. 1A). Weight loss was positively correlated with fat loss, indicating that exercise-induced weight loss is mainly due to fat mass loss (Fig. 1B).

Effect of the 8-week exercise program on serum leptin and adiponectin levels. To assess whether fat mass loss reduced the serum levels of adipokine, which is predominantly produced in adipose tissue, the leptin and adiponectin levels were measured prior to and following the program (Fig. 2). Serum leptin levels (mean \pm SEM) were significantly decreased following the program (from $9.82 \pm 0.98$ to $7.23 \pm 0.67 \mathrm{ng} / \mathrm{ml}$ ), and were significantly correlated with body fat percentage $(r=0.6093$, $\mathrm{P}<0.0001)$. Serum adiponectin levels were unchanged and were significantly negatively correlated with body fat percentage $(\mathrm{r}=-0.4774, \mathrm{P}=0.0032)$. The results demonstrated that serum leptin levels were more strongly correlated with body fat percentage than with serum adiponectin levels, which is also controlled by other physiological factors (Fig. 2).

Effect of the 8-week exercise program on serum osteopontin $(O P N)$ levels. To determine whether serum OPN levels were significantly correlated with body fat percentage, the levels were measured prior to and following the 8-week body weight control program (Fig. 3A). Serum OPN levels (mean \pm SEM) were significantly decreased following the program (from $16.03 \pm 2.34$ to $10.65 \pm 1.22 \mathrm{ng} / \mathrm{ml}$ ). However, serum OPN levels were not correlated with body fat percentage in the 18 subjects who completed the 8-week program, suggesting that serum OPN levels are controlled by other factors in humans (Fig. 3B).
In addition, serum OPN levels of subjects with a body fat percentage of $>33 \%$ were negatively correlated with body fat percentage $(\mathrm{r}=-0.5332, \mathrm{P}<0.05)$ (Fig. 3C).

\section{Discussion}

We hypothesized that serum OPN levels are decreased by exercise-induced fat mass loss and are associated with body fat percentage. As additional and indirect tests of the hypothesis, the association of body fat percentage with adipokines, serum leptin and adiponectin, which are mainly produced in adipose tissues, were also determined. Thus, it was demonstrated that serum leptin levels were positively correlated with body fat percentage, while serum adiponectin levels were negatively correlated with body fat percentage. By contrast, serum OPN levels were not correlated with body fat percentage, regardless of the fact that serum OPN levels decreased following 8 weeks of exercise-induced weight loss. Furthermore, serum OPN levels were negatively associated with body fat percentage in subjects with a body fat percentage of $>33 \%$ (Fig. 3C). This indicated that serum OPN levels were not correlated with body fat percentage and may be affected by other tissues and physiological conditions.

Obesity is regarded as a state of systemic, chronic, low-grade inflammation. It is considered to be a risk factor associated with the genesis or development of various diseases, including coronary heart disease, hypertension, type 2 diabetes mellitus, cancer, respiratory complications and osteoarthritis (20-22). Exercise-induced weight loss is regarded as the safest method to prevent obesity-related diseases. Thus, there has been interest in clarifying how physical activity and exercise modulate obesity-mediated inflammation (23). The anti-inflammatory effects of regular exercise may be mediated via a reduction in the visceral fat mass (with a subsequent decreased release of adipokines) and the induction of an anti-inflammatory environment with each exercise session (24).

Exercise-induced weight loss resulted in a decrease in serum leptin levels and no change in serum adiponectin levels in this study. Similarly, even mild weight loss induced by calorie restriction has been suggested to have beneficial effects on serum leptin levels in humans; however, it has no clear impact on serum adiponectin levels (25). This observation suggested that leptin is mainly produced in adipose tissues; the serum leptin concentration was increased in obese patients and was correlated with body fat percentage in the present study, as previously demonstrated (26). However, serum adiponectin levels were not altered following weight loss in the present 

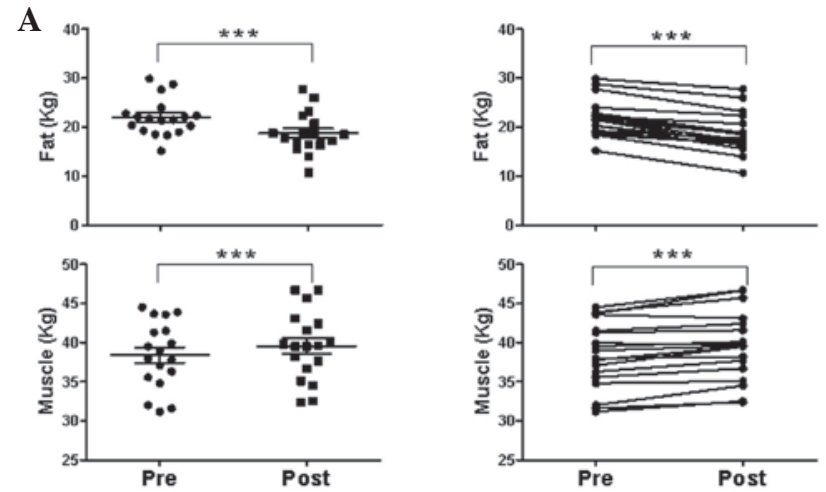

B

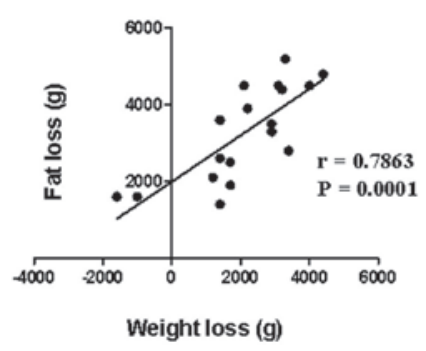

Figure 1. Effect of the 8-week exercise program on fat and muscle mass. (A) The change in fat and muscle mass prior to and following the exercise program. (B) The association between weight loss and fat loss. Fat and muscle mass were measured in 18 subjects following the 8 -week exercise program. The values are expressed as the mean \pm SEM. Data were analyzed using the Wilcoxon signed-rank test (two-tailed). The degree of linearity between the two variables was compared using the Spearman's correlation test (two-tailed). ${ }^{* * *} \mathrm{P}<0.0001$.
A
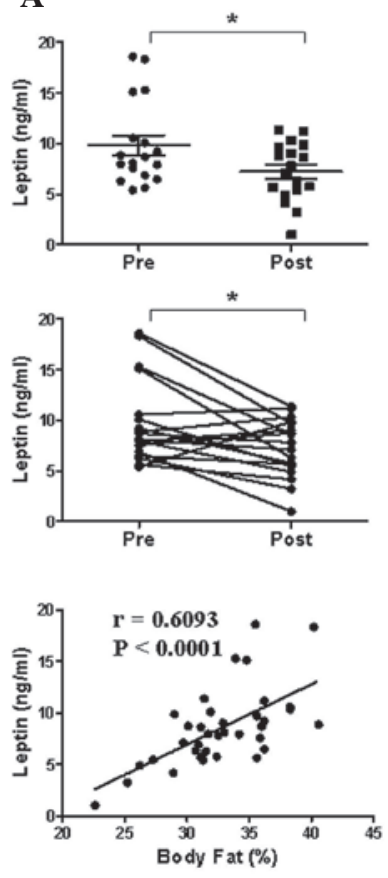

в
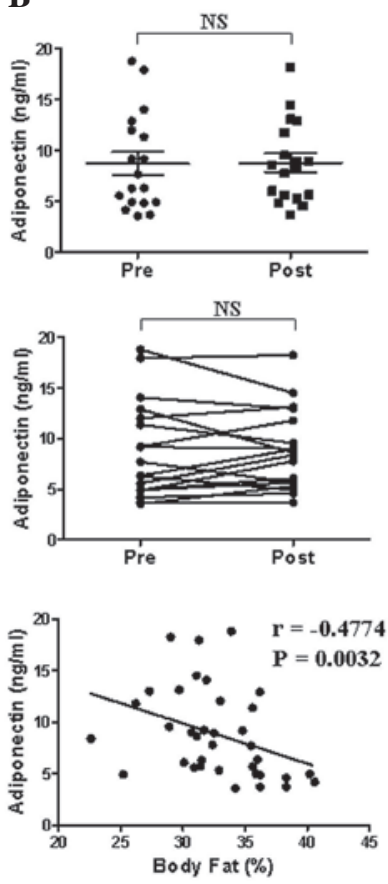

Figure 2. Effect of the 8-week exercise program on serum (A) leptin and (B) adiponectin levels. Serum leptin and adiponectin levels were measured in 18 subjects following the 8-week exercise program. The values are expressed as the mean \pm SEM. Data were analyzed as described in Fig. 1. ${ }^{*}<<0.05$.

study, but were negatively correlated with body fat percentage, although adiponectin is also highly expressed in adipose
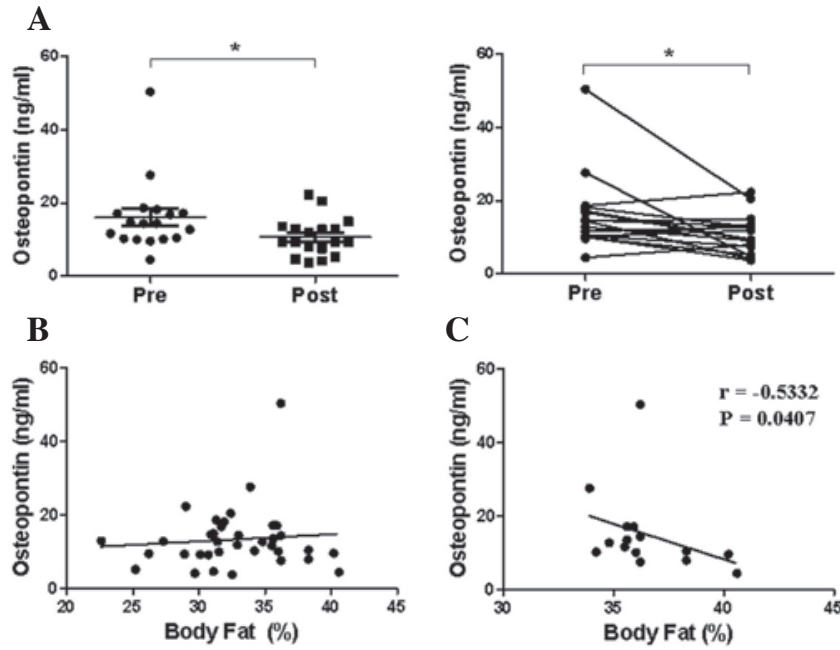

Figure 3. Effect of the 8-week exercise program on serum osteopontin (OPN) levels. (A) Serum OPN levels were measured in 18 subjects following the 8-week exercise program. (B) No correlation between serum OPN levels and body fat percentage was demonstrated. (C) A negative correlation between serum OPN levels and body fat percentage was observed in subjects with $>33 \%$ body fat. The values are expressed as the mean \pm SEM. Data were analyzed as described in Fig. 1 . $^{*} \mathrm{P}<0.05$.

tissues (27). Unlike leptin, serum adiponectin levels appear to be regulated by unknown physiological factors. Adiponectin levels are inversely proportional to obesity, diabetes and other insulin-resistant states (28). Therefore, in obesity, reducing chronic adipose tissue inflammation and macrophage infiltration may be beneficial for reversing the downregulation of adiponectin gene expression by pro-inflammatory cytokines (29). Serum OPN levels also appear to be associated with fat mass. OPN is extensively upregulated in the adipose tissue of obese humans, as well as in that of diet-induced and genetically $(\mathrm{db} / \mathrm{db})$ obese mice. However, there is controversy concerning the correlation between serum OPN concentrations and fat mass in humans and mice. Serum OPN concentrations remained unchanged in murine models of obesity, although OPN was highly upregulated in the adipose tissue of high-fat diet-induced and genetically obese mice (4). By contrast, in another study, obese and overweight patients exhibited significantly increased circulating OPN concentrations as compared with lean subjects (obese, $72.6 \pm 28.5 \mathrm{ng} / \mathrm{ml}$; overweight, $68.2 \pm 20.8 \mathrm{ng} / \mathrm{ml}$; lean, $42.7 \pm 27.9 \mathrm{ng} / \mathrm{ml} ; \mathrm{P}<0.001$ ) (5). The results of the present study showed that serum OPN levels may also be affected by other physiological factors as opposed to body fat percentage alone. For example, serum OPN levels appear to be affected by age. Riedl et al demonstrated that there is a weak, but significant, negative correlation between OPN levels and age (30). A possible correlation between OPN and age-related changes in bone mineral density (BMD) was hypothesized. Thus, only college students (mean age, 20.7 \pm 0.4 years) were recruited in the present study to exclude the age factor. No significant difference was identified in serum OPN levels between males and females (30). In another study, whole body vibration was demonstrated to decrease serum OPN levels, an effect that appears to be associated with the change in bone metabolism (31). Thus, in our study, the reduction of serum OPN levels may have resulted from body vibrations which may 
be triggered by excercise, as opposed to exercise-induced fat loss. This observation is due to the fact that OPN is a component of bone matrix and is important in bone turnover, serving as an anchor for osteoclasts and thus activating the resorption cascade (18). Exercise may also be correlated with the change in bone metabolism (32), as physical activity has the potential to reduce the risk of osteoporotic fractures. Extreme inactivity may cause rapid bone loss of $\leq 40 \%$, while athletic activity results in bone hypertrophy of $\leq 40 \%$ (32). The mechanisms for the beneficial effect of exercise on bone mass appear to be due to a cell response to hormonal and mechanical load stimuli.

Furthermore, dietary components are known to affect the gene expression and plasma concentration of adiponectin in humans and animals (33). Animal models have demonstrated that the consumption of hyperlipidemic diets, rich in saturated fat, reduces the levels of adiponectin, while diets rich in polyunsaturated fatty acids supplemented with $\Omega 3$ and eicosapentaenoic acid increase its gene expression and plasma levels. In humans, the consumption of a healthy and Mediterranean diet is positively correlated with adiponectin levels, although the mechanisms have not been elucidated (33). Exercise and dietary components may also have affected serum OPN levels in the present study, as a specific/calorie-restricted diet was not implemented. It has been demonstrated that OPN expression in cardiomyocytes was significantly correlated with the impaired function of the left ventricle, which was the main source of circulating OPN plasma levels (34). Furthermore, adipokines is involved either directly or indirectly in the regulation of bone remodeling (35); the change in serum leptin level induced by exercise may induce the change of bone remodeling and the change of bone metabolism may affect the serum OPN levels. In addition, calorie restriction-induced weight loss appears to be a risk factor for rapid bone loss. However, physical activityinduced weight loss preserves BMD (36). In conclusion, serum OPN levels may be regulated by various physiological factors. Thus, the elevated expression of OPN in adipose tissues may not be correlated with serum OPN levels. Instead, other tissues or physiological factors may have a greater contribution to serum OPN levels as compared to fat mass. Thus, the correlation between serum OPN levels and body fat loss remains to be elucidated.

\section{Acknowledgements}

This study was supported by the Basic Science Research Program through the National Research Foundation of Korea (NRF) and funded by the Ministry of Education, Science and Technology (Korea) (grant nos. 2012-0002659 and 2011-0026939).

\section{References}

1. Harford KA, Reynolds CM, McGillicuddy FC and Roche HM: Fats, inflammation and insulin resistance: insights to the role of macrophage and T-cell accumulation in adipose tissue. Proc Nutr Soc 70: 408-417, 2011.

2. Morimoto J, Kon S, Matsui Y and Uede T: Osteopontin; as a target molecule for the treatment of inflammatory diseases. Curr Drug Targets 11: 494-505, 2010.

3. Nomiyama T, Perez-Tilve D, Ogawa D, et al: Osteopontin mediates obesity-induced adipose tissue macrophage infiltration and insulin resistance in mice. J Clin Invest 117: 2877-2888, 2007
4. Kiefer FW, Zeyda M, Todoric J, et al: Osteopontin expression in human and murine obesity: extensive local up-regulation in adipose tissue but minimal systemic alterations. Endocrinology 149: 1350-1357, 2008.

5. Gómez-Ambrosi J, Catalán V, Ramírez B, et al: Plasma osteopontin levels and expression in adipose tissue are increased in obesity. J Clin Endocrinol Metab 92: 3719-3727, 2007.

6. Xu G, Sun W, He D, et al: Overexpression of osteopontin in rheumatoid synovial mononuclear cells is associated with joint inflammation, not with genetic polymorphism. J Rheumatol 32: 410-416, 2005

7. Braitch M and Constantinescu CS: The role of osteopontin in experimental autoimmune encephalomyelitis (EAE) and multiple sclerosis (MS). Inflamm Allergy Drug Targets 9: 249-256, 2010.

8. Frenzel DF and Weiss JM: Osteopontin and allergic disease: pathophysiology and implications for diagnostics and therapy. Expert Rev Clin Immunol 7: 93-109, 2011.

9. Waller AH, Sanchez-Ross M, Kaluski E and Klapholz M Osteopontin in cardiovascular disease: a potential therapeutic target. Cardiol Rev 18: 125-131, 2010.

10. Naldini A, Leali D, Pucci A, et al: Cutting edge: IL-1beta mediates the proangiogenic activity of osteopontin-activated human monocytes. J Immunol 177: 4267-4270, 2006.

11. Rangaswami H, Bulbule A and Kundu GC: Osteopontin: role in cell signaling and cancer progression. Trends Cell Biol 16: 79-87, 2006.

12. Kiefer FW, Neschen S, Pfau B, et al: Osteopontin deficiency protects against obesity-induced hepatic steatosis and attenuates glucose production in mice. Diabetologia 54: 2132-2142, 2011.

13. Zeyda M, Gollinger K, Todoric J, et al: Osteopontin is an activator of human adipose tissue macrophages and directly affects adipocyte function. Endocrinology 152: 2219-2227, 2011.

14. Komorowski J, Jankiewicz-Wika J, Kolomecki K, et al: Systemic blood osteopontin, endostatin, and E-selectin concentrations after vertical banding surgery in severely obese adults. Cytokine 55: 56-61, 2011.

15. Schaller G, Aso Y, Schernthaner GH, et al: Increase of osteopontin plasma concentrations after bariatric surgery independent from inflammation and insulin resistance. Obes Surg 19: 351-356, 2009.

16. Shevde LA, Das S, Clark DW and Samant RS: Osteopontin: an effector and an effect of tumor metastasis. Curr Mol Med 10: 71-81, 2010

17. Giachelli CM and Steitz S: Osteopontin: a versatile regulator of inflammation and biomineralization. Matrix Biol 19: 615-622, 2000.

18. Reinholt FP, Hultenby K, Oldberg A and Heinegård D: Osteopontin - a possible anchor of osteoclasts to bone. Proc Natl Acad Sci USA 87: 4473-4475, 1990.

19. Friedewald WT, Levy RI and Fredrickson DS: Estimation of the concentration of low-density lipoprotein cholesterol in plasma, without use of the preparative ultracentrifuge. Clin Chem 18: 499-502, 1972.

20. Sowers MR and Karvonen-Gutierrez CA: The evolving role of obesity in knee osteoarthritis. Curr Opin Rheumatol 22: 533-537, 2011.

21. Shehzad A, Ha T, Subhan F and Lee YS: New mechanisms and the anti-inflammatory role of curcumin in obesity and obesityrelated metabolic diseases. Eur J Nutr 50: 151-161, 2011.

22. Trayhurn P and Wood IS: Adipokines: inflammation and the pleiotropic role of white adipose tissue. Br J Nutr 92: 347-355, 2004.

23. Wärnberg J, Cunningham K, Romeo J and Marcos A: Physical activity, exercise and low-grade systemic inflammation. Proc Nutr Soc 69: 400-406, 2010.

24. Gleeson M, Bishop NC, Stensel DJ, et al: The anti-inflammatory effects of exercise: mechanisms and implications for the prevention and treatment of disease. Nat Rev Immunol 11: 607-615, 2011.

25. Klempel MC and Varady KA: Reliability of leptin, but not adiponectin, as a biomarker for diet-induced weight loss in humans. Nutr Rev 69: 145-154, 2011.

26. Frühbeck G, Gómez-Ambrosi J, Muruzábal FJ and Burrell MA: The adipocyte: a model for integration of endocrine and metabolic signaling in energy metabolism regulation. Am J Physiol Endocrinol Metab 280: E827-E847, 2001.

27. Arita Y, Kihara S, Ouchi N, et al: Paradoxical decrease of an adipose-specific protein, adiponectin, in obesity. 1999. Biochem Biophys Res Commun 31: 560-564, 2012. 
28. Shehzad A, Iqbal W, Shehzad O and Lee YS: Adiponectin: regulation of its production and its role in human diseases. Hormones (Athens) 11: 8-20, 2012

29. Guerre-Millo M: Adiponectin: an update. Diabetes Metab 34: 12-18, 2008.

30. Riedl M, Vila G, Maier C, et al: Plasma osteopontin increases after bariatric surgery and correlates with markers of bone turnover but not with insulin resistance. J Clin Endocrinol Metab 93: 2307-2312, 2008.

31. Humphries B, Fenning A, Dugan E, et al: Whole-body vibration effects on bone mineral density in women with or without resistance training. Aviat Space Environ Med 80: 1025-1031, 2009.

32. Smith EL and Gilligan C: Physical activity effects on bone metabolism. Calcif Tissue Int 49 (Suppl): S50-S54, 1991.
33. Reis CE, Bressan J and Alfenas RC: Effect of the diet components on adiponectin levels. Nutr Hosp 25: 881-888, 2010.

34. Tamura A, Shingai M, Aso N, et al: Osteopontin is released from the heart into the coronary circulation in patients with a previous anterior wall myocardial infarction. Circ J 67: 742-744, 2003.

35. Oh KW, Lee WY, Rhee EJ, et al: The relationship between serum resistin, leptin, adiponectin, ghrelin levels and bone mineral density in middle-aged men. Clin Endocrinol (Oxf) 63: 131-138, 2005.

36. Villareal DT, Fontana L, Weiss EP, et al: Bone mineral density response to caloric restriction-induced weight loss or exerciseinduced weight loss: a randomized controlled trial. Arch Intern Med 166: 2502-2510, 2006. 